\title{
Patterns of daily energy drink consumption differ by gender among Belgian adolescents
}

\section{Abstract}

Over the past decade, energy drinks (ED) have become increasingly popular among adolescents. Due to their high content in caffeine, sugar and other energy-promoting components, frequent ED consumption may have adverse health effects, like cardiovascular symptoms, sleep impairment, and nervousness. Previous studies have shown that ED consumption was more frequent among males. To extend this knowledge, our analysis examined how sociodemographic and lifestyle determinants of frequent ED consumption may differ by gender among Belgian adolescents. This study was based on data self-reported by 9,119 12-19 year-old French-speaking Belgian adolescents in secondary school, within the 2014 cross-sectional Health Behaviour in School-Aged Children survey. Pupils reporting they usually consumed ED once a day or more were considered as "high ED consumers". Sociodemographic and lifestyle characteristics were described in relation to ED consumption, for boys and girls separately. We used multiple logistic regression models, stratified by gender, to assess the associations between the sociodemographic and lifestyle variables, with high ED consumption. Daily ED consumption was reported by $7.9 \%$ of boys and $4.6 \%$ of girls. In both genders, the proportion of high ED consumers was significantly higher among adolescents of grades 1-3, those of lower family affluence, in first- and second-generation immigrants, those watching TV more than 6 hours/day, those reporting later bedtimes, and those consuming soft drinks daily. In girls only, higher percentages were observed in girls from single- $(7.6 \%)$ or blended-families $(5.2 \%)$, than in girls from two-parent families $(3.5 \%)$. In boys only, this percentage was higher among adolescents meeting physical activity recommendations (14.5\% vs $6.5 \%$ ). Multivariate analyses showed that high ED consumption was associated with lower grades, long screen time, later bedtimes, and daily soft drink consumption in both genders. In girls only, adolescents from single-parent families were more likely to consume ED daily (OR 1.70; 95\% CI 1.23-2.37) (vs. two-parent families). In boys only, first-generation immigrants (OR 1.53; 95\% CI 1.06-2.21) (vs. natives) and youth sufficiently physically active (OR 2.19; 95\% CI 1.65-2.90) were more likely to consume ED daily. High ED consumption was more frequent among boys and showed gender-specific patterns, with associations with family structure in girls only, and with migration status and physical activity in boys only. Our findings provide evidence about patterns of high ED consumption among adolescents and should be used to inform the development of prevention strategies.

\section{Conflict of Interest}

There is no conflict of interest. 